\title{
On the Zeros of Daubechies Orthogonal and Biorthogonal Wavelets
}

\author{
Jalal Karam \\ Faculty of Science and General Studies, Alfaisal University, Riyadh, KSA \\ Email: jkaram@alfaisal.edu
}

Received February 3, 2012; revised April 27, 2012; accepted May 4, 2012

\begin{abstract}
In the last decade, Daubechies' wavelets have been successfully used in many signal processing paradigms. The construction of these wavelets via two channel perfect reconstruction filter bank requires the identification of necessary conditions that the coefficients of the filters and the roots of binomial polynomials associated with them should exhibit. In this paper, orthogonal and Biorthogonal Daubechies families of wavelets are considered and their filters are derived. In particular, the Biorthogonal wavelets Bior3.5, Bior3.9 and Bior6.8 are examined and the zeros distribution of their polynomials associated filters are located. We also examine the locations of these zeros of the filters associated with the two orthogonal wavelets $d b 6$ and $d b 8$.
\end{abstract}

Keywords: Orthogonal; Biorthogonal Wavelets; Binomial Polynomials

\section{Introduction}

The Daubechies wavelets construction, requires the finding of a scaling function $\phi(t)$ and a wavelet function $\psi(t)$ [1]. This construction is best described via a two-channel perfect reconstruction filter bank [1,2] and depends on the distribution of the zeros of some polynomials in the plane. The literature provides many theorems describing geometric locations of the roots of certain polynomials $[3,4]$. Identifying necessary conditions for the coefficients of the filters associated with the construction is vital for orthogonality, Biorthogonality and alias cancellation. The distribution of the zeros of the binomial polynomial related to the construction of these wavelets were proved to reside inside the unit circle [5], and better limits for these roots based on a generalization of the Kakeya-Enestrom Theorem were derived in [6] where it was shown that if $y$ is a root of a binomial polynomial of degree $p-1$, then: $\frac{1}{2 p} \leq|y| \leq \frac{1}{2}$. A subclass of polynomials is derived from this construction process by considering the ratios of consecutive binomial polynomials' coefficients essentials to these constructions. We showed mathematically that the roots of this class of polynomials reside inside the unit circle. In Section 3, the construction of Daubechies orthogonal wavelets is detailed. In Section 4, the new class of polynomials is in-

"Dedication: This paper is dedicated to my professors and supervisors Karl Dilcher and William Phillips. troduced and the distribution of their zeros is examined and mathematically shown to reside inside the unit circle. An example is then presented illustrating the location for the zeros of the derived polynomial of the orthogonal mother wavelet $d b 6$. The case of $d b 8$ is then examined in Section 5 and results are obtained. Section 6 describe the conclusion of this work.

\section{Related Works}

A two-channel filter bank has a low-pass and a high-pass filter in the decomposition (analysis) phase and anotherlow-pass and a high-pass filter in the reconstruction (synthesis) phase. Let $H_{0}$ and $G_{0}$ denote the low-pass filter coefficients and the high-pass filter coefficients respectively of the analysis phase, then given the coefficients of $H_{0}$, it is shown in [1,7] that the coefficients of the filters $H_{1}, G_{0}$ and $G_{1}$ that lead to orthogonality can easily be derived from the coefficients of $H_{0}$. Therefore, to construct a Daubechies orthogonal wavelet, all we need to do is to find the coefficients of the filters $H_{0}$ associated with it.

The distribution of the zeros of a family of polynomials having their coefficients as the ratios of those of the binomial polynomials is considered in Section 4 and proved to reside inside the unit circle. Similar discussions about Daubechies' Biorthogonal wavelets family are also included along with the constructions of Bior3.5, Bior3.9 and Bior6.8. In the orthogonal case, the scaling and wavelet functions are derived from the coefficients of the 
filters $H_{0}, H_{1}, G_{0}$ and $G_{1}$. They must satisfy respectively the following two equations [1]:

$$
\begin{aligned}
& \phi(t)=2 \sum_{k=0}^{l-1} h_{1}(k) \phi(2 t-k) \\
& W(t)=2 \sum_{k=0}^{l-1} g_{1}(k) \phi(2 t-k)
\end{aligned}
$$

Biorthogonal filter banks produce Biorthogonal wavelets. This calls for a new scaling function $\tilde{\phi}(t)$ and a new wavelet function $\tilde{w}(t)$. Here, one needs the following conditions: $H_{1}(z)=H_{0}^{-1}(z)$ and $G_{1}(z)=H_{1}^{-1}(z)$ [8]. The wavelet filters for analysis banks are derived [1] from the scaling filters using the two relations:

$$
\begin{aligned}
& h_{1}=(-1)^{n+1} g_{0}(n) \\
& g_{1}=(-1)^{n} h_{0}(n)
\end{aligned}
$$

The analysis scaling and wavelet equations thus become:

$$
\begin{gathered}
\tilde{\phi}(t)=\sum_{0}^{\tilde{N}} 2 h_{0}^{r}(k) \tilde{\phi}(2 t-k) \\
\tilde{w}(t)=\sum_{0}^{N} 2 g_{0}^{r}(k) \tilde{\phi}(2 t-k)
\end{gathered}
$$
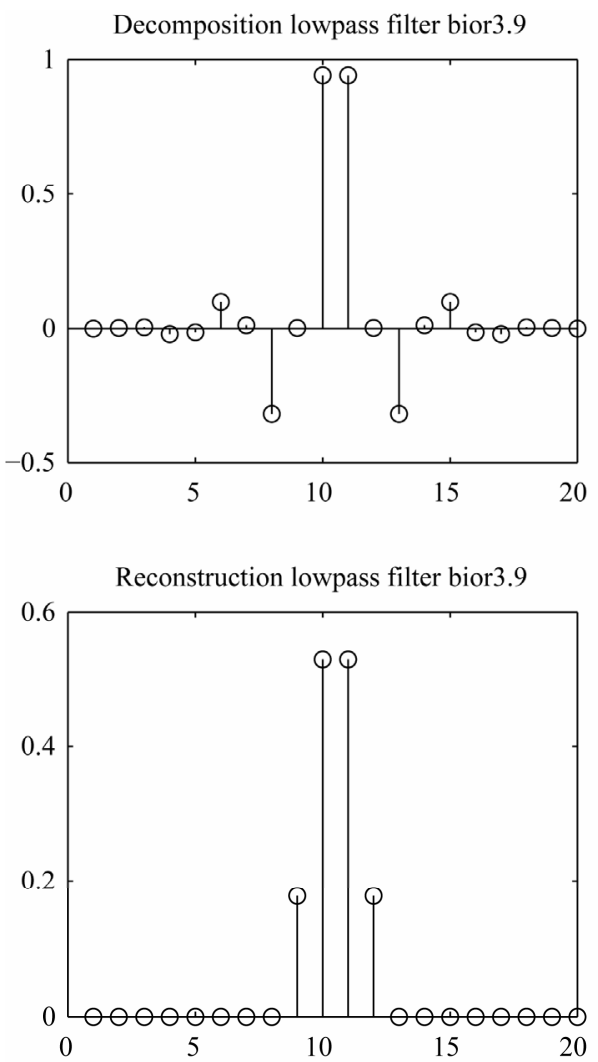

where $h_{0}^{r}$ and $g_{0}^{r}$ are the reverse of the original filters $h_{0}$ and $g_{0}$ respectively. The construction of $\phi(t), w(t)$, $\tilde{\phi}(t)$ and $\tilde{w}(t)$ starts with imposing the Biorthogonality conditions on the filters. The lowpass analysis coefficients $h_{0}^{r}(k)$ are the product of a double shift Biorthogonal to the lowpass synthesis coefficients $h_{1}(k)$ :

$$
\begin{aligned}
& 2 \sum h_{1}(k) h_{0}^{r}(k+2 n)=\delta(n) \\
& 2 \sum g_{1}(k) g_{0}^{r}(k+2 n)=\delta(n)
\end{aligned}
$$

And the highpass filter is Biorthogonal to the lowpass filter:

$$
\begin{aligned}
& \sum h_{1}(k) g_{0}^{r}(k+2 n)=0 \text { and } \\
& \sum g_{1}(k) h_{0}^{r}(k+2 n)=0
\end{aligned}
$$

Figure 1 shows the frequency responses of the decomposition and reconstruction filters and, the decomposition and reconstruction scaling and wavelet functions of the Biorthogonal (Bior3.9) [9]. Figure 2 shows this Biorthogonl wavelet zeros' distribution of its decomposition and reconstruction filters. This wavelet possesses the propreties of being smooth with a linear phase and short length filters. Also, Table 1 displays the coefficients of the low-passes and high-passes filters of Bior3.9. Figure $\mathbf{3}$ and Figure $\mathbf{4}$ show the zeros' distributions for the filters associated with Bior6.8 and Bior3.5 respectively.
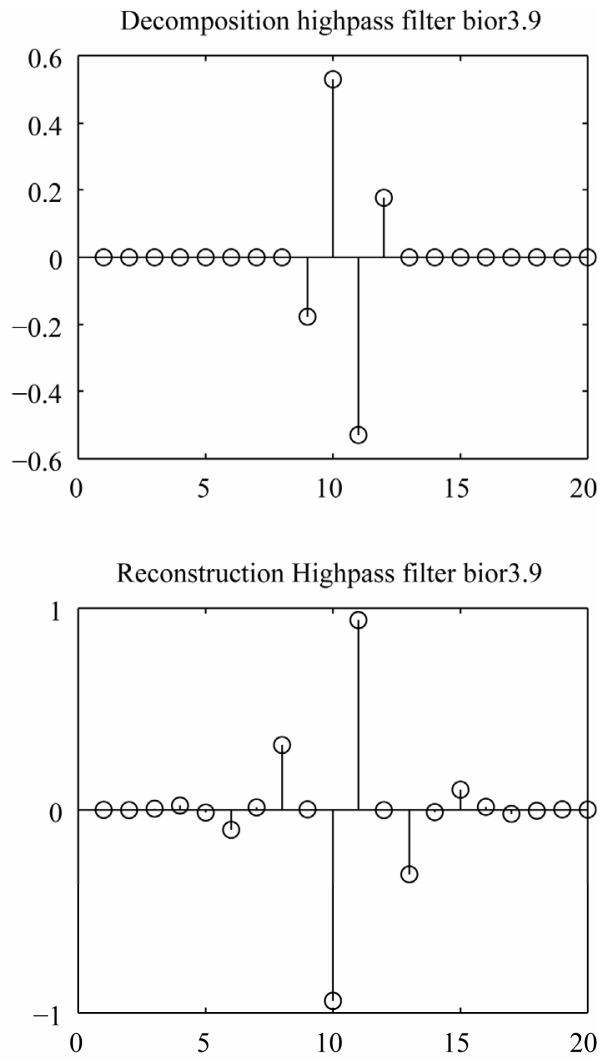

Figure 1. Bior3.9 impulse response for the decomposition and reconstruction filters. 

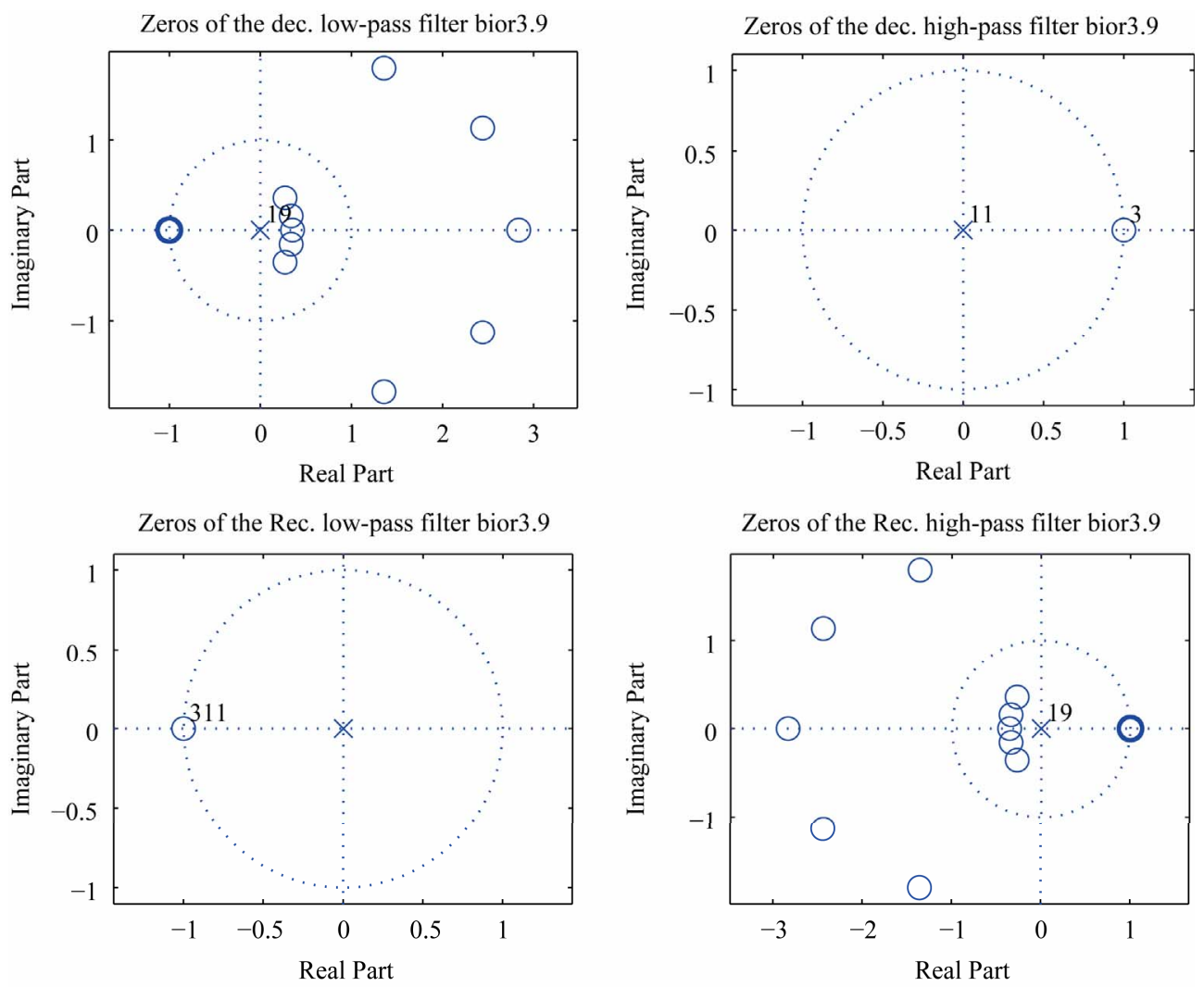

Figure 2. Bior3.9 zeros distribution of the decomposition and reconstruction filters.
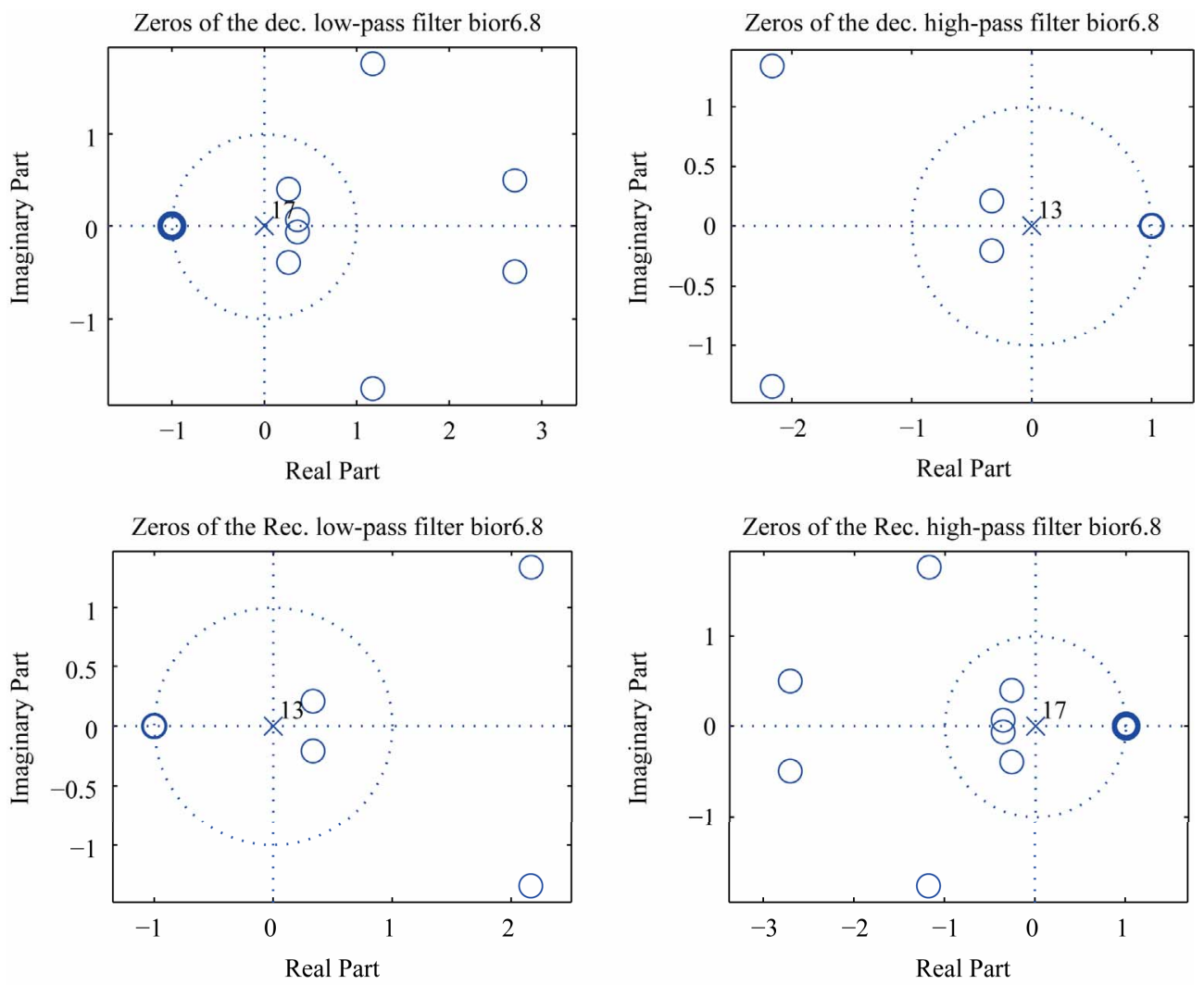

Figure 3. Bior6.8 zeros distribution of the decomposition and reconstruction filters. 

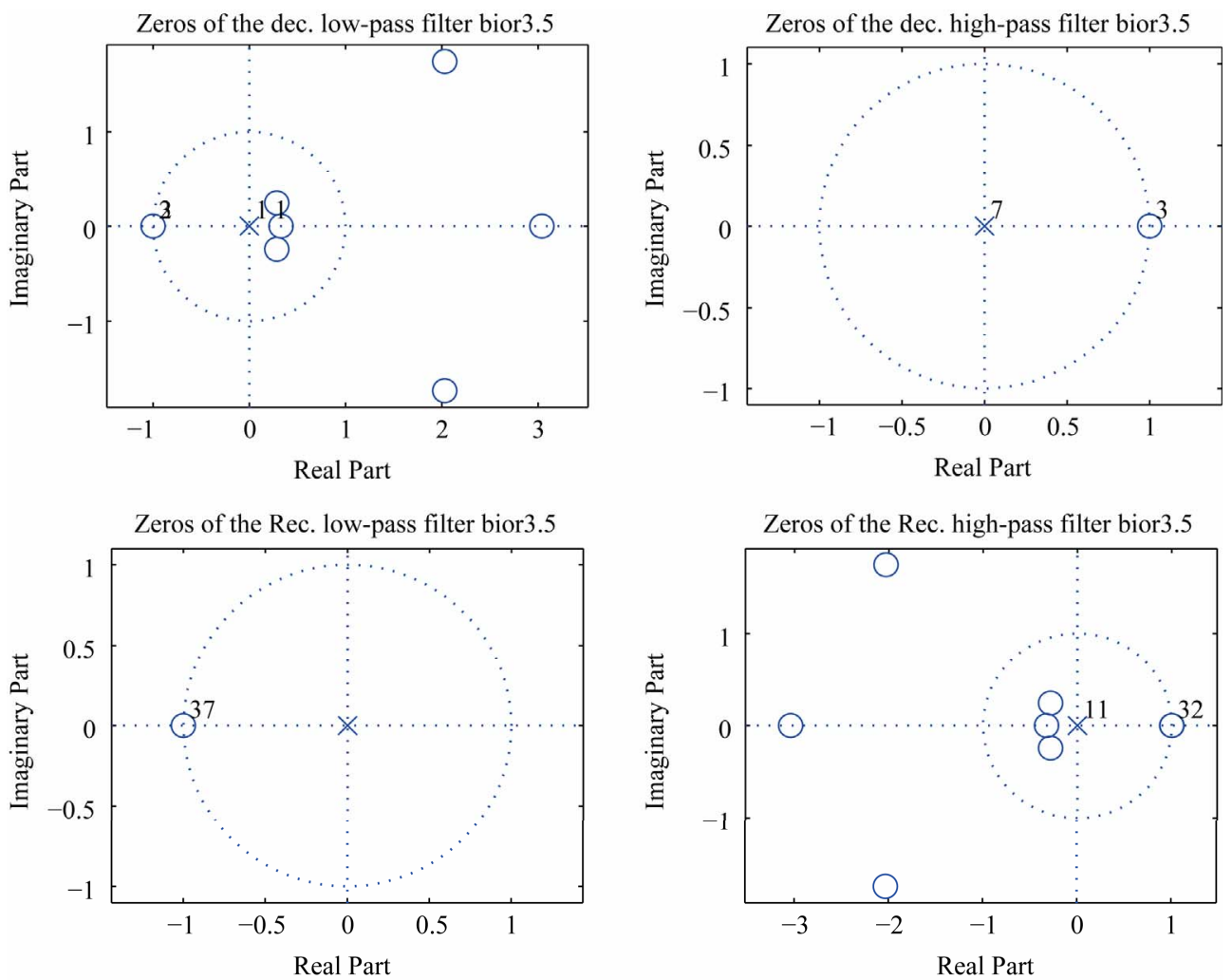

Figure 4. Bior3.5 zeros distribution of the decomposition and reconstruction filter.

Table 1. Filter coefficients of the biorthogonal wavelet Bior3.9.

\begin{tabular}{cccc}
\hline$H_{0}$ & $H_{1}$ & $\tilde{H}_{0}$ & $\tilde{H}_{1}$ \\
\hline-0.0007 & 0 & 0 & -0.0007 \\
0.0020 & 0 & 0 & -0.0020 \\
0.0051 & 0 & 0 & 0.0051 \\
-0.0206 & 0 & 0 & 0.0206 \\
-0.0141 & 0 & 0 & -0.0141 \\
0.0991 & 0 & 0 & -0.0991 \\
0.0123 & 0 & 0 & 0.0123 \\
-0.3202 & 0 & 0 & 0.3202 \\
0.0021 & -0.1768 & 0.1768 & 0.0021 \\
0.9421 & 0.5303 & 0.5303 & -0.9421 \\
0.9421 & -0.5303 & 0.5303 & 0.9421 \\
0.0021 & 0.1768 & 0.1768 & -0.0021 \\
-0.3202 & 0 & 0 & -0.3202 \\
0.0123 & 0 & 0 & -0.0123 \\
0.0991 & 0 & 0 & 0.0991 \\
-0.0141 & 0 & 0 & 0.0141 \\
-0.0206 & 0 & 0 & -0.0206 \\
0.0051 & 0 & 0 & -0.0051 \\
0.0020 & 0 & 0 & 0.0020 \\
-0.0007 & 0 & 0 & 0.0007 \\
\hline
\end{tabular}

\section{Construction of Daubechies Orthogonal Wavelets}

Wavelets such as $d b 6$ and $d b 8$ have played a very essential role in a variety of speech recognition and compression paradigms introduced last decade [10,11]. The low-pass and high-pass filters in the decomposition phase of a two channel filter bank are depicted in Figure 5 and two more filters of the reconstruction phase are displayed in Figure 6.

Let $h_{0}(n)$ and $g_{0}(n)$ denote the low-pass filter coefficients and the high-pass filter coefficients respectively in the analysis phase. To obtain perfect reconstruction, these two filters must satisfy the following conditions [1,2]:

1) For the low-pass filter $h_{0}(n)$ :

$$
\begin{aligned}
& \sum_{n} h_{0}(n)=1 \quad \sum_{n} h_{0}^{2}(n)=1 / 2 \\
& 2 \sum_{n} h_{0}(n) h_{0}(n-2 k)=\delta(k)
\end{aligned}
$$

2) For the high-pass filter $g_{0}(n)$ :

$$
\begin{aligned}
& \sum_{n} g_{0}(n)=1 \sum_{n} g_{0}^{2}(n)=1 / 2 \\
& 2 \sum_{n} g_{0}(n) g_{0}(n-2 k)=\delta(k)
\end{aligned}
$$

where $\delta(k)$ is the Dirac delta function defined by: 
Input Signall

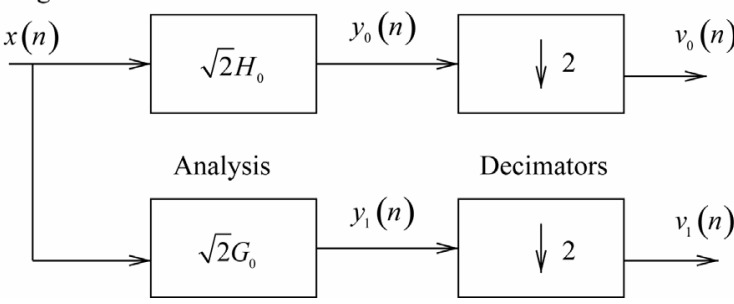

Figure 5. Analysis phase filters of a two-channels filter bank.

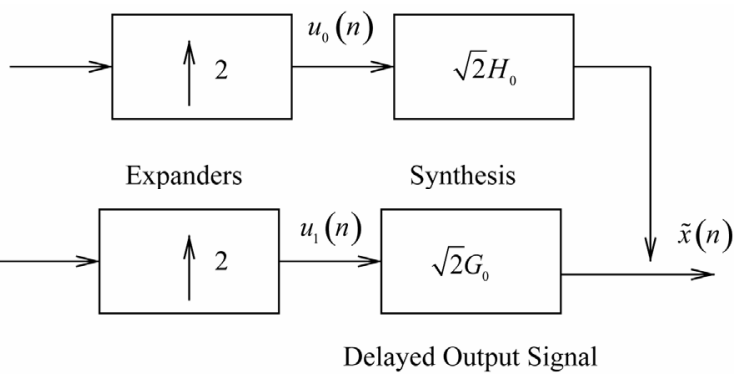

Figure 6. Synthesis phase filters of a two-channels filter bank.

$$
\delta(k)= \begin{cases}1 & \text { if } k=0 \\ \text { or } & \\ 0 & \text { otherwise. }\end{cases}
$$

Given the coefficients of $h_{0}$, it is shown in $[1,7]$ that the coefficients of the filters $h_{1}(n), g_{0}(n)$ and $g_{1}(n)$ that lead to orthogonality can easily be derived from the coefficients of $h_{0}$. Therefore, to construct a Daubechies orthogonal wavelet, all we need to do, is to find the coefficients of the filter $h_{0}$ associated with it.

The construction of the filter bank amounts to [1]:

1) Design a product low-pass filter $P_{0}$ satisfying:

$$
P_{0}(z)-P_{0}(-z)=2 z^{-l}
$$

2) Factor $P_{0}$ in $H_{1} H_{0}$, then find $G_{1}$ and $G_{0}$. And can be reduced even further by defining:

$P(z)=z^{l} P_{0}(z)$ and substituting $P(-z)$ by $-z^{l} P_{0}(-z)$. Hence, the perfect reconstruction condition becomes [8]:

$$
P(z)+P(-z)=2
$$

which implies that $P(z)$ is a half band filter [1] with all of its the coefficients zeros except the constant term 1. Furthermore, the odd powers cancel when we add $P(z)$ to $P(-Z)$. The design of the low-pass and the high-pass filters of the synthesis and analysis filter banks of a Daubechies orthogonal wavelet, considers the following two properties [1]:

1) These wavelets filters must be orthogonal.

2) And must have maximum flatness at $w=0$ and $w=\pi$ in their frequency responses.
The low-pass filters will have $p$ zeros at $\pi$, and have a total of $2 p$ coefficients, (length of the filters). This filter bank is orthogonal and the product filters $P_{0}(z)$ and $P_{1}(z)$ have a length of $4 p-2$. The construction of Daubechies orthogonal wavelets begins by choosing the number of zeros $p$ at $\pi$. The zeros the filters associated with the $d b 6$ are depicted in Figure 7. Here, we also need to choose the binomial polynomial $B_{p}(y)$ associated with it which has a degree of $p-1$. The coefficients of these polynomials can be found recursively for $p$ by using the following equation:

$$
b(i)=b(i+1) * \frac{(2 p-i-1)}{4 *(p-i)}
$$

For a given value $p$, the coefficients of $B_{p}(y)$ are in an ascending order [5]. To get the roots of $B_{p}(y)$, one scales $b$ by 4 and to facilitate the numerical calculations, one uses the variable $4 y$ instead of $y$. The ratio of any two consecutive coefficients is:

$$
r_{k}=\frac{b_{k}}{b_{k+1}}=\frac{(p+k-2) !}{(p-1) ! *(k-1) !} * \frac{(p-1) ! * k !}{(p+k-1) !}
$$

Which in its simplest form can be expressed as:

$$
r_{i}=\frac{i-1}{i+p}, i=0,1,2, \cdots, p-2
$$

This equation will be used in Section 5 to construct the family of polynomials with coefficients equal to the ratios of this polynomial consecutive coefficients.

Now to compute the $2 p-2$ zeros of $P(z)$ other than -1 , we note that according to $[1,9]$ the frequency response of the half-band filter $P(w)$ is given by:

$$
P(w)=2(1-y)^{p} B(y)
$$

where $\cos (w)=1-2 y$ or $y=(1-\cos (w)) / 2$.

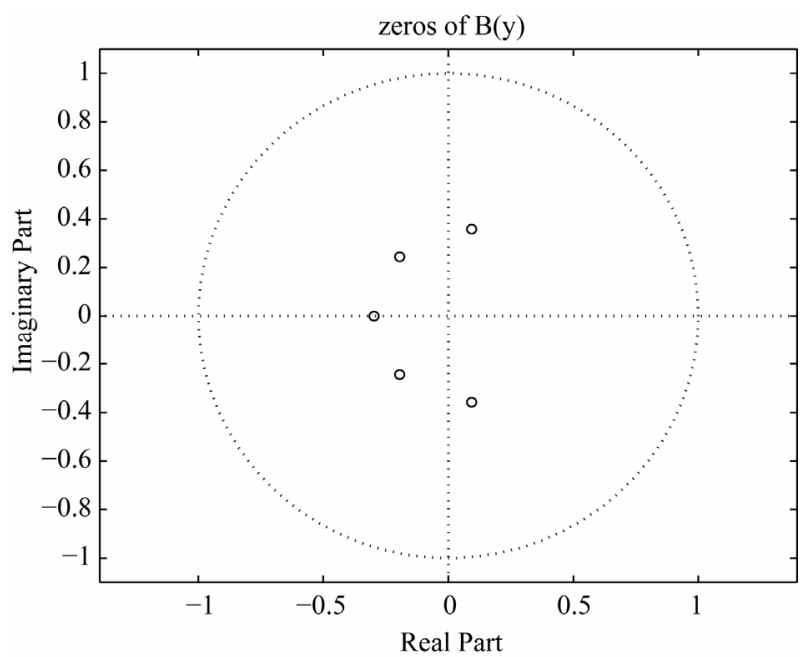

Figure 7. Zeros of $B(y)$ for $d b 6$. 
On the unit circle we have:

$$
(z+1 / z) / 2=\cos (w)=1-2 y .
$$

Also, off the unit circle we use the same relation between $z$ and $y$. Rearranging these terms leads to:

$$
(1 / z)\left(z^{2}-2(1-2 y) z+1\right)=0
$$

Now let $x=1-2 y$ and $u=\sqrt{\left(x^{2}-1\right)}$ then,

$$
(1 / z)\left(z^{2}-2 x z+1\right)=0
$$

with $z \neq 0$, this implies that:

$$
z=x+u
$$

and

$$
z=x-u
$$

are the two roots of $P(z)$ for each root $y$ of $B(y)$.

Note that $x-u=\frac{1}{x+u}$.

That is, we have $p-1$ roots and their inverses, namely:

$$
x=1-2 * y
$$

and

$$
u=\sqrt{x^{2}-1}
$$

and

$$
z=[x+u, x-u] .
$$

The distribution of these zeros in the plane is shown in Figure 8. From $P(z), P_{0}(z)$ is then derived and all is left is to factorize $P_{0}(z)$. Daubechies did the following factorization found in [1]:

$$
P_{0}(z)=\left(\frac{1+z^{-1}}{2}\right)^{2 p} Q_{2 p-2}(z)
$$

where $Q_{2 p-2}(z)$ is a polynomial of degree $2 p-2$.

\section{The Construction of $\mathbf{d b 6}$}

For $p=6$, the $d b 6$ wavelet is obtained. Figure 8 shows the location in the complex plane for the zeros of $B_{6}(y)$ associated with the Daubechies $d b 6$ orthogonal wavelet. The frequency responses of the analysis lowpass filter $H_{0}(z)$ and synthesis lowpass filter $H_{1}(z)$ of this wavelet are depicted in Figure 9. Therefore completing the construction of the scaling function along with the mother wavelet. The decomposition and reconstruction functions for the mother wavelet $d b 6$ are plotted in Figure 9, while Figure 10 shows the impulse response of the four filters associated with it.

Now, given the coefficients $h(0), h(1), \cdots, h(n-1)$ of the low-pass filter $h_{0}$, it is shown in $[1,7]$ that the coefficients of the filters $h_{1}(n), g_{0}(n)$ and $g_{1}(n)$ that
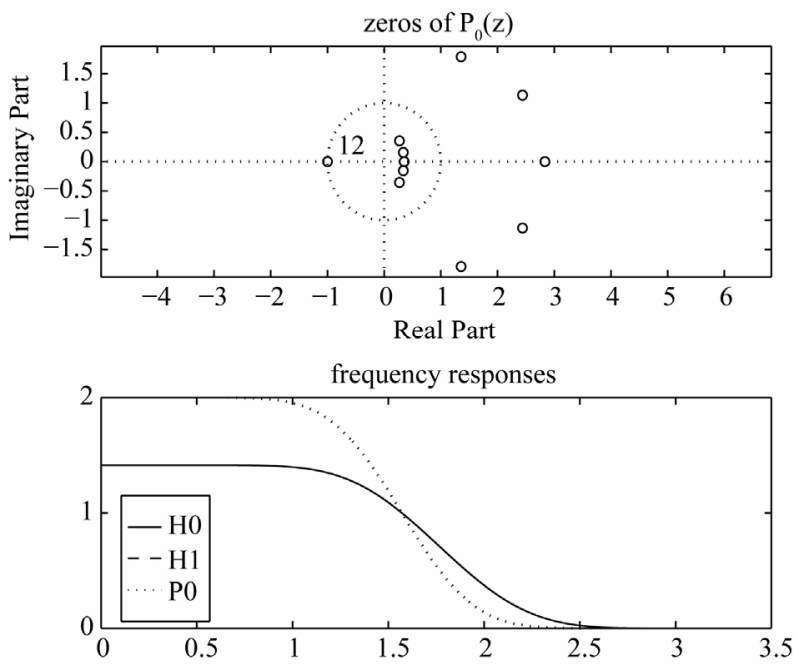

Figure 8. Zeros of $P(z)$ for $d b 6$ and the frequency responses of the filters $P, H_{0}$ and $H_{1}$.

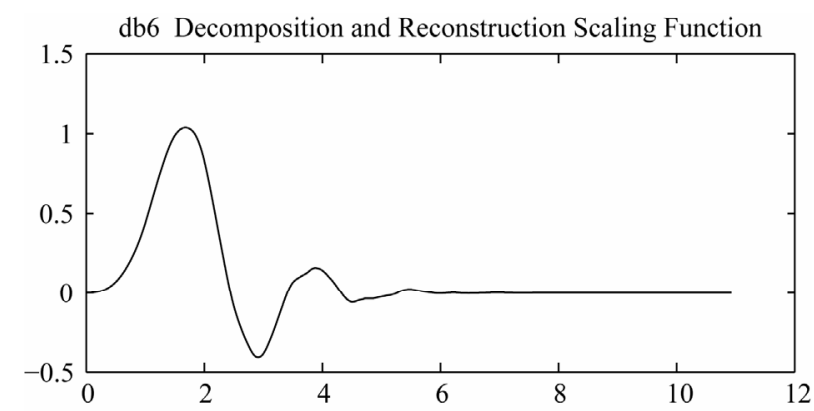

db6 Wavelet Decomposition and Reconstruction Wavelet Function

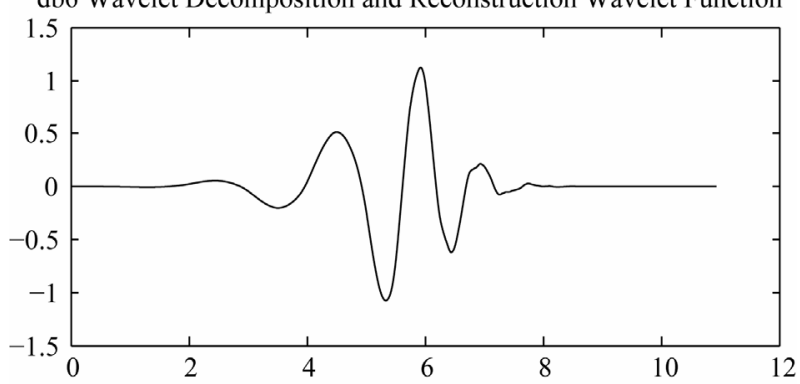

Figure 9. The $d b 6$ analysis and synthesis scaling and wavelet functions.

lead to orthogonality can be derived from the coefficients of $h_{0}$ as follows:

First, the coefficients of the high-pass filter $g_{0}(n)$ of the analysis bank are obtained from those of the low-pass filter $h_{0}(n)$ by the "alternating flip".

This can be represented by three operations on the coefficients of $h_{0}(n)$.

1) Reverse the order;

2) Alternate the signs;

3) Shift by an odd number $l$.

This takes the low-pass filter coefficients into an orthogonal high-pass filter [1] which is represented in the 

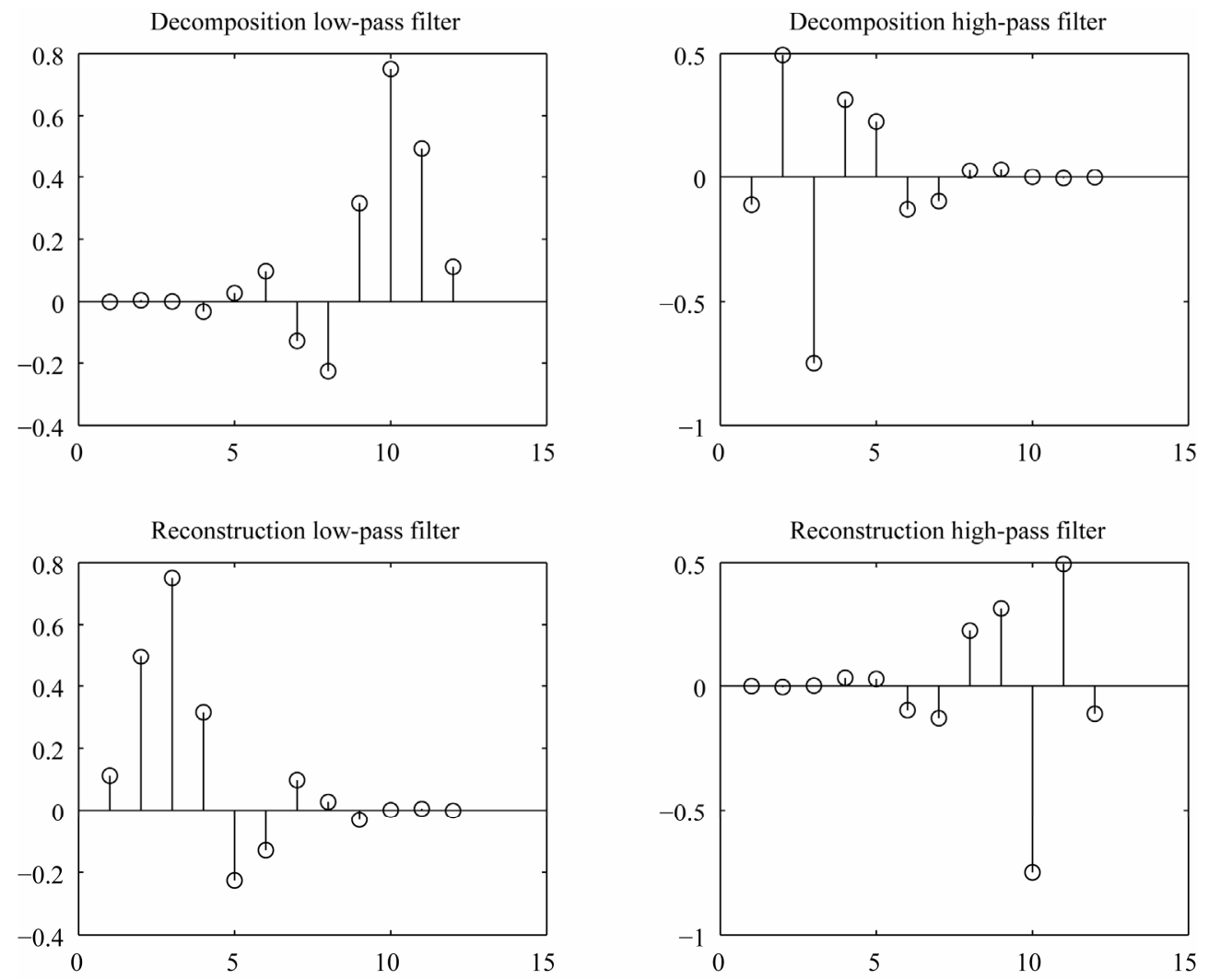

Figure 10. Impulse response for the reconstruction and decomposition filters of $\boldsymbol{d b 6}$.

following equation:

$$
g_{0}(n)=(-1)^{n} h_{0}(l-n)
$$

Then, the coefficients of the high-pass filter $g_{1}(n)$ of the synthesis bank are obtained by the reverse of the coefficients of the high-pass filter $g_{0}(n)$ of the analysis bank. They can be generated by the following equation:

$$
g_{1}(n)=h_{0}(l-n)
$$

The coefficients of the low-pass filter $g_{1}(n)$ of the synthesis bank are the alternating flip of the coefficients of $g_{0}(n)$. They can be generated by the following equation:

$$
h_{1}(n)=(-1)^{n} g_{1}(l-n)
$$

where $l$ is the length of the low-pass filter $h_{0}(n)$ [1].

The scaling and wavelet functions are then derived from the coefficients of these filters. The scaling function satisfies the equation [1]:

$$
\phi(t)=2 \sum_{k=0}^{l-1} h_{1}(k) \phi(2 t-k)
$$

where, $h_{1}(k)$ is the reverse of $h_{0}(k)$ and the wavelet function is then derived from the scaling function by the equation:

$$
W(t)=2 \sum_{k=0}^{l-1} g_{1}(k) \phi(2 t-k)
$$

\section{Zeros of Ratio Coefficients Polynomials}

Now we consider the class of polynomials with coefficients those of the ratios obtained in Equation (4). An optimal limit of these zeros in the complex plane was presented in [12]. Other theorems and alternative approach to proving this distribution can be found in [13]. Now the rations can be expressed as follows:

$$
\begin{aligned}
Q_{p-2}(z)= & \frac{-1}{p}+0 \cdot z^{1}+\frac{1}{2+p} z^{2} \\
& +\frac{3}{4+p} z^{3}+\cdots+\frac{p-2}{2 p-2} z^{p-2} .
\end{aligned}
$$

We note that these coefficients are in an ascending order where $a_{k}-a_{k-1}>0$.

Theorem I: The roots of the polynomials $Q_{p-2}$ lie inside the unit disk for all $p$.

Proof: For $a_{0}=-\frac{1}{p}<0$, consider

$$
(1-z) Q(z)=\phi(z)-a_{p-2} z^{p-1}
$$

where 


$$
\phi(z)=a_{0}+\sum_{k=1}^{p-2}\left(a_{k}-a_{k-1}\right) z^{k} .
$$

For $z \neq 0$, we have:

$$
z_{p-2} \phi\left(\frac{1}{z}\right)=a_{0} z^{p-2}+\sum_{k=1}^{p-2}\left(a_{k}-a_{k-1}\right) z^{p-2-k} .
$$

Now for $|z| \leq 1$,

$$
\begin{aligned}
\left|z^{p-2} \phi\left(\frac{1}{z}\right)\right| & \leq\left|a_{0}\right|+\sum_{k=1}^{p-2}\left(a_{k}-a_{k-1}\right) \\
& \leq\left|a_{0}\right|-a_{0}+a_{p-2} .
\end{aligned}
$$

and

$$
\left|\phi\left(\frac{1}{z}\right)\right| \leq \frac{\left|a_{0}\right|-a_{0}+a_{p-2}}{\left|z^{p-2}\right|} .
$$

Replacing $z$ with $\frac{1}{Z}$, we get:

$$
\phi(z) \leq\left(\left|a_{0}\right|-a_{0}+a_{p-2}\right)\left|z^{p-2}\right| \text { for } z \neq 0 .
$$

Hence, if $|z|>\frac{\left(\left|a_{0}\right|-a_{0}+a_{p-2}\right)}{\left|a_{p-2}\right|}$ (i.e. $\left.|z| \geq 1\right)$, then:

$$
\begin{aligned}
|(1-z) Q(z)| & =\left|\phi(z)-a_{p-2} z^{p-1}\right| \\
& \geq\left|a_{p-2}\right||z|^{p-1}-|\phi(z)| \\
& \geq\left|a_{p-2}\right|\left|z^{p-1}\right|-\left(\left|a_{0}\right|-a_{0}+a_{p-2}\right)|z|^{p-2} \\
& >0
\end{aligned}
$$

\section{The Distributions of Zeros for $\mathbf{d b 6}$}

For the Daubechies wavelet " $d b 6$ " this polynomial is:

$$
Q_{4}(z)=-1 / 6+0+1 / 8 z+2 / 9 z^{2}+3 / 10 z^{3}+4 / 11 z^{4}
$$

with maximum module of 0.9325 . The roots of this polynomial are depicted below in Figure 11 and observed to reside all in the unit circle.

\section{The Construction of $d b 8$}

The scaling and wavelet functions are then derived from the coefficients of these filters. They satisfy respectively the equations [1]:

$$
\begin{aligned}
& \phi(t)=2 \sum_{k=0}^{l-1} h_{1}(k) \phi(2 t-k) \\
& W(t)=2 \sum_{k=0}^{l-1} g_{1}(k) \phi(2 t-k)
\end{aligned}
$$

To compute the 14 zeros of $P(z)$ other than -1 , note that on and off the unit circle we have the following relation between $z$ and $y:(z+1 / z) / 2=1-2 y$. This implies that the equation:

$$
(1 / z)\left(z^{2}-2(1-2 y) z+1\right)=0 .
$$

The coefficients of $B_{8}(y)$ are displayed in Table 2. If one sets $x=1-2 y$ and $u=\sqrt{x^{2}-1}$ then, $z=x+$ $u$ and $z=x-u$ are the two roots of $P(z)$ for each root $y$ of $B_{8}(y)$. Note that $x-u=1 /(x+u)$. That is, we have 7 roots and their inverses, The plot of these zeros in the plane are shown in Figure 12. Also, the values of $|x|,|y|$ and $|u|$ are listed in Table 3.

From the definition of $P(z), P_{0}(z)$ is obtained and all is left now is to factorize $P_{0}(z)$. Were $Q_{14}(z)$ is a polynomial of degree 14 and chosen to satisfy Equation 16. It is equal to $B_{14}(y)$. The different factorizations of $P_{0}(z)$ into $H_{0}(z) * H_{1}(z)$ lead to different mother wavelets. Choosing $H_{0}(z)$ to have its seven zeros inside the unit circle and $H_{1}(z)$ to have its seven zeros outside the unit circle leads to the Daubechies orthogonal mother wavelet $d b 8$.

The scaling and wavelet functions of one of the Daubechies wavelets' family member called $d b 8$ are shown in Figure 13. The same figure also shows the impulse response of the four filters associated with that wavelet and Table 4 displays The coefficients of the of $d b 8$ filters.

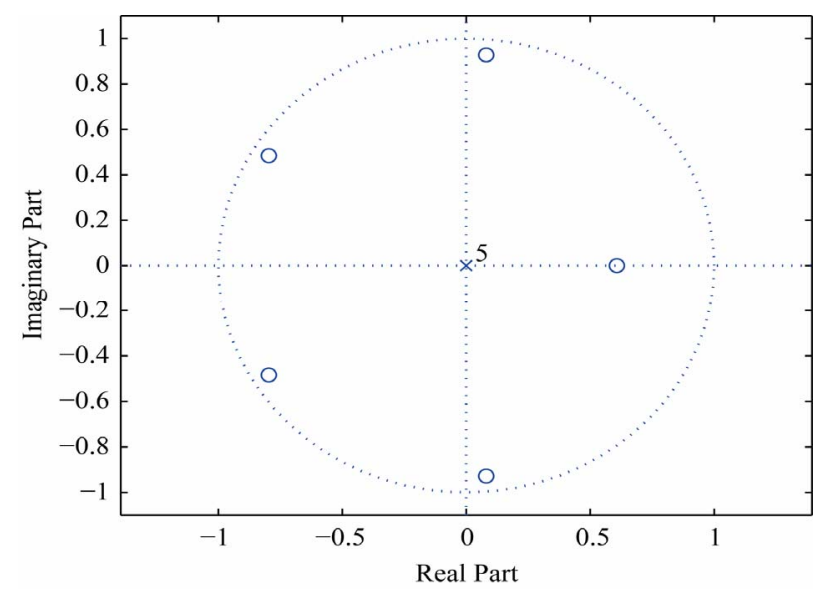

Figure 11. Zeros of $Q_{4}(z)$ for Daubechies $d b 6$ orthogonal wavelet.

Table 2. The coefficients of the polynomial $B_{8}(y)$.

\begin{tabular}{ll}
\hline$b(1)$ & 1.0000 \\
$b(8)$ & 0.2095 \\
$b(7)$ & 0.4189 \\
$b(6)$ & 0.7734 \\
$b(5)$ & 1.2891 \\
$b(4)$ & 1.8750 \\
$b(3)$ & 2.000 \\
$b(2)$ & 2.2500 \\
\hline
\end{tabular}


Table 3. The roots of $Q_{14}(z)$ inside the unit circle $z=x-u$ and outside of it $z=x+u$.

\begin{tabular}{ccccc}
\hline$|u|$ & $|x|$ & $|y|$ & $|z|>1$ & $|z|<1$ \\
\hline 1.1466 & 0.9187 & 0.3712 & 2.0179 & 0.4956 \\
1.1466 & 0.9187 & 0.3712 & 2.0179 & 0.4956 \\
1.1716 & 1.2852 & 0.3094 & 2.4246 & 0.4124 \\
1.1716 & 1.2852 & 0.3094 & 2.4246 & 0.4124 \\
1.1856 & 1.5511 & 0.2755 & 2.7367 & 0.3654 \\
1.1824 & 1.4860 & 0.2832 & 2.6592 & 0.3761 \\
1.1824 & 1.4860 & 0.2832 & 2.6592 & 0.3761 \\
\hline
\end{tabular}

Table 4. The coefficients of the of $d b 8$ filters.

\begin{tabular}{cc}
\hline$H_{0}$ & $H_{1}$ \\
\hline-0.0001 & 0.0544 \\
0.0007 & 0.3129 \\
-0.0004 & 0.6756 \\
-0.0049 & 0.5854 \\
0.0087 & -0.0158 \\
0.0140 & -0.2840 \\
-0.0441 & 0.0005 \\
-0.0174 & 0.1287 \\
0.1287 & -0.0174 \\
0.0005 & -0.0441 \\
-0.2840 & 0.0140 \\
-0.0158 & 0.0087 \\
0.5854 & -0.0049 \\
0.6756 & -0.0004 \\
0.3129 & 0.0007 \\
0.0544 & -0.0001 \\
\hline
\end{tabular}

To carry out the factorization we note that $P(z)$ has 16 roots at $z=-1$ and 14 other roots which occur in pairs $(z$ and $1 / z)$. This means that we have 7 roots inside the unit circle and the other 7 roots outside the unit circle. The roots inside the circle are the roots for the filter $H_{0}(z)$ coming from the equation: $z=x-u$ and the ones outside it are for filter $H_{1}(z)$ obtained from the equation: $z=x+u$. These roots when factorized lead to the coefficients of these two filters and they are shown in Table 3 for $Q_{14}(z)$. The coefficients of the high-pass filters $G_{0}(z)$ and $G_{1}(z)$ are simply then derived from the low-pass filter coefficients by the alternating sign property.

The scaling and wavelet functions of one of the
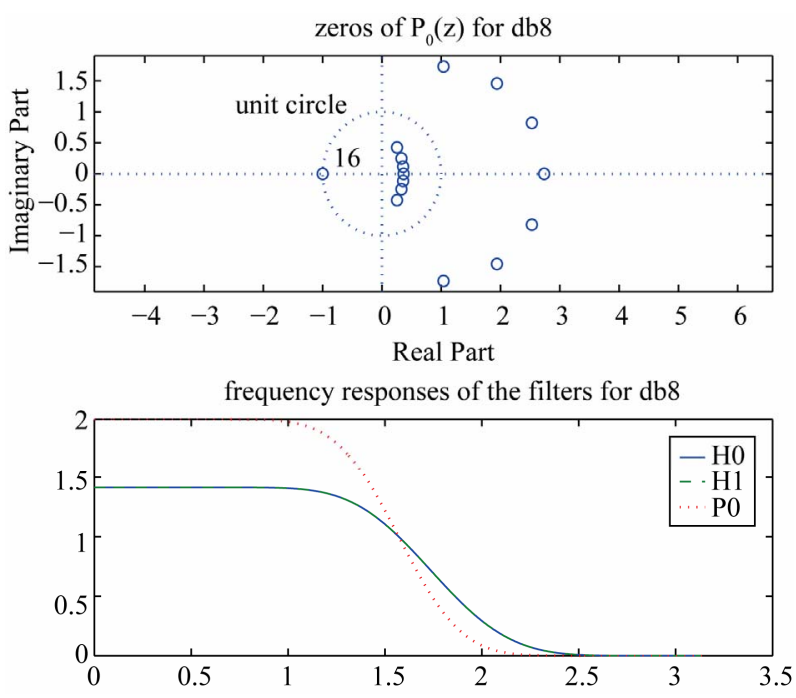

Figure 12. Zeros of $P(z)$ for $d b 8$ and the frequency responses of the filters: $P_{0}, H_{0}$ and $H_{1}$.

Daubechies wavelets' family member called $d b 8$ are shown in Figure 13. The same figure also shows the impulse response of the four filters associated with that wavelet.

The general characteristics of this wavelet include compact support for which exact reconstruction are possible with FIR filters. Its associated scaling filter is a minimum-phase filter. This wavelet is a member of the orthogonal set of wavelets that are usually denoted by: $d b(N) \quad N$ represents the order of the reconstruction and decomposition wavelet. Their corresponding filter length is $2 * N$.

\section{Conclusion}

In this paper we construct Daubechies orthogonal wavelets via the two channel perfect reconstruction filter bank. The cases of $d b 6$ and $d b 8$ are examined where we derived the coefficients of the filters associated with these wavelets and the roots of the binomial polynomials that made this construction possible. The locations of the zeros of the polynomials involved in this construction were found and their locations were discussed. The distribution of the zeros of a family of polynomials having their coefficients as the ratios of those of the binomial polynomials was examined and were proved to reside inside the unit circle. Similar discussions about the Daubechies Biorthogonal wavelets family are included along with the constructions of Bior3.5, Bior3.9 and Bior6.8.

\section{Acknowledgements}

The author would like to thank Alfaisal University and its Office of Research for securing the time, environment and funds to complete this research project. This work 


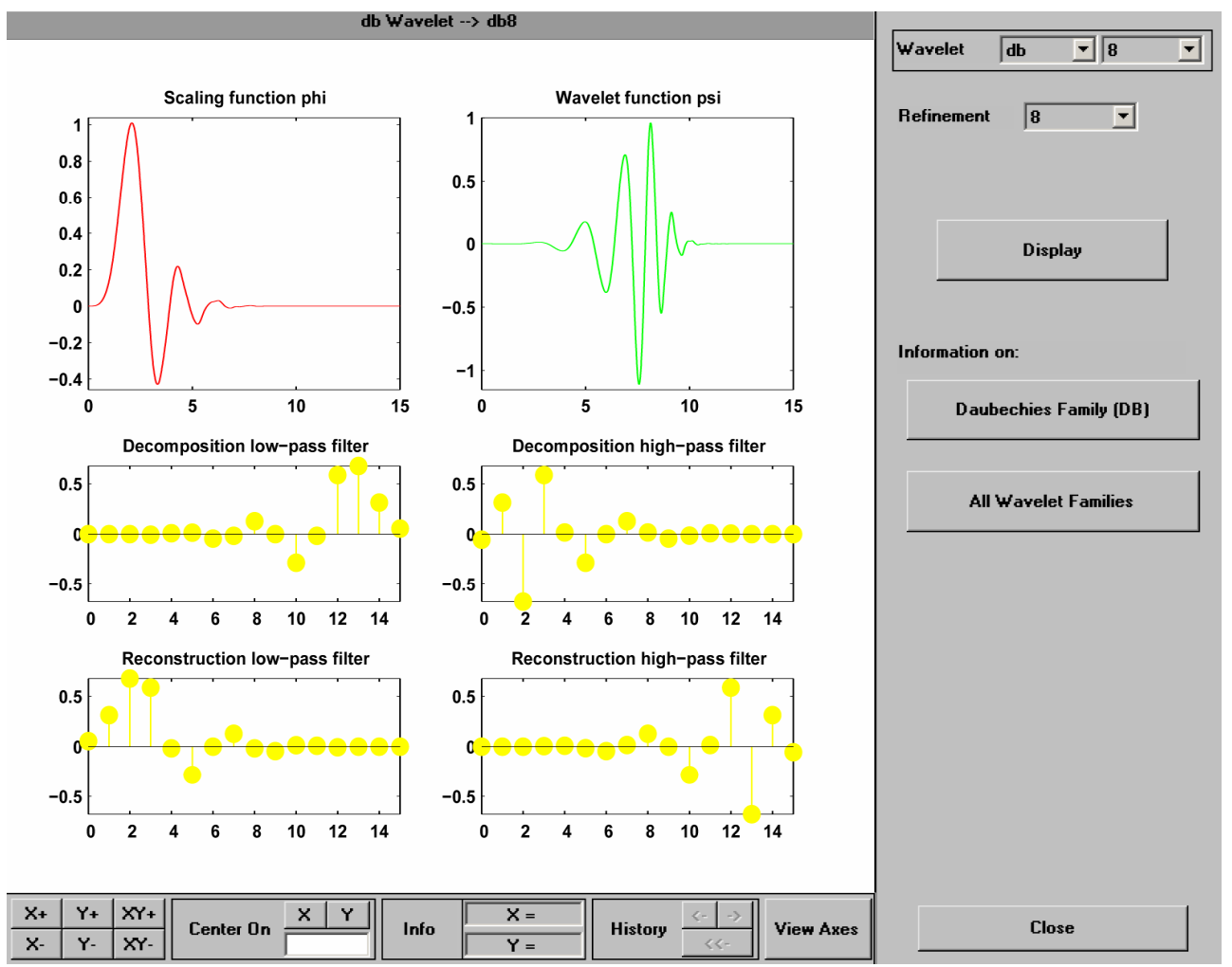

Figure 13. $d b 8$ Wavelet and scaling functions. The impulse response for the reconstruction and decomposition filters of the wavelet $d b 8$.

was also supported by the Alfaisal University Start-Up Fund (No. 410111410091).

\section{REFERENCES}

[1] G. Strang, and T. Nguyen, "Wavelets and Filter Banks," Wellesley-Cambridge Press, Wellesley, 1996.

[2] I. Daubechies, "Ten Lectures on Wavelets," SIAM, Philadelphia, 1992.

[3] S. Kakeya, "On the Limits of the Roots of an Algebraic Equation with Positive Coefficients," Tohoku Mathematical Journal, Vol. 2, 1912, pp. 140-142.

[4] J. Karam, "On the Kakeya-Enestrom Theorem," MSc. Thesis, Dalhousie University, Halifax, 1995.

[5] J. Karam, "Connecting Daubechies Wavelets with the Kakeya-Enestrom Theorem," International Journal of Applied Mathematics, Vol. 14, No. 2, 2003, pp. 109-124.

[6] J. Karam, "On the Roots of Daubechies Polynomials," International Journal of Applied Mathematics, Vol. 20, No. 8, 2007, pp. 1069-1076.

[7] M. Vetterli, "Wavelets and Filter Banks: Theory and De- sign," IEEE Transactions on Signal Processing, Vol. 40, No. 9, 1992, pp. 2207-2232. doi:10.1109/78.157221

[8] M. Vetterli and J. Kovacevic, "Wavelets and Suband Coding," Prentice Hall, Englewood Cliffs, 1995.

[9] M. Misiti, Y. Misiti, G. Oppenheim and J. Poggi, "Matlab Wavelet Tool Box,” 1997.

[10] J. Karam, "A Comprehensive Approach for Speech Related Multimedia Applications," WSEAS Transactions on Signal Processing, Vol. 6, No. 1, 2010, pp. 12-21.

[11] J. Karam, "Radial Basis Functions With Wavelet Packets For Recognizing Arabic Speech," The 9th WSEAS International Conference on Circuits, Systems, Electronics, Control and Signal Processing, Athens, December 2010, pp. 34-39.

[12] J. Karam, "On the Distribution of Zeros for Daubechies Orthogonal Wavelets and Associated Polynomials," 15th WSEAS International Conference on Applied Mathematics, Athens, 29-31 December 2010, pp. 101-105.

[13] C. A. Muresan, "Comparative Methods for the Polynomial Isolation," Proceedings of the 13th WSEAS International Conference on Computers, 2009, pp. 634-638. 